\title{
EXACT SOLUTIONS FOR NATURAL CONVECTION OF LAYERED FLOWS OF A VISCOUS INCOMPRESSIBLE FLUID WITH SPECIFIED TANGENTIAL FORCES AND THE LINEAR DISTRIBUTION OF TEMPERATURE ON THE LAYER BOUNDARIES
}

\author{
N. V. Burmasheva ${ }^{1,2}$ *, E. Yu. Prosviryakov ${ }^{1}$ \\ ${ }^{I}$ Institute of Engineering Science, Urals Branch, Russian Academy of Sciences, \\ 34, Komsomolskaya st., Ekaterinburg, 620049, Russian Federation \\ ${ }^{2}$ Ural Federal University named after the First President of Russia B. N. Yeltsin, \\ 19, Mira st., Ekaterinburg, 620002, Russian Federation \\ *Corresponding author. E-mail: nat burm@mail.ru \\ Address for correspondence: 34, Komsomolskaya St., Ekaterinburg, 620049, Russian Federation \\ Tel.:+7 (343) 37535 76; fax: +7 (343) 3745330
}

\begin{abstract}
A new exact solution of the Oberbeck-Boussinesq equations for the convective flow of a viscous incompressible fluid is considered. Layered flows of a viscous incompressible fluid are investigated within the class of the Sidorov-Lin exact solutions, which generalizes the family of the Ostroumov-Birikh solutions. The use of an exact solution allows an overdetermined system of fluid motion equations to be solved. The fluid is heated by setting a heat source at both boundaries. The dimension of the studied boundary value problem cannot be lowered by the transformation of the rotation. The obtained exact solution describes the counterflow in the fluid. Thermocline and a boundary layer occur near one of the boundary layers in the fluid flow.
\end{abstract}

Keywords: layered flow, exact solution, counterflows.

DOI: $10.17804 / 2410-9908.2017 .4 .016-031$

\section{References}

1. Joseph D.D. Stability of fluid motions. Berlin, Heidelberg, New York, Springer-Verlag, 1976.

2. Gershuni G.Z., Zhukhovitskii E.M. Convective Stability of Incompressible Fluids. Israel Program for Scientific Translations. Jerusalem, Keter Publishing House, 1976, 330 p.

3. Shtern V. Counterflows. Paradoxical Fluid Mechanics Phenomena. Cambridge University Press, 2012, 469 p. DOI: 10.1017/CBO9781139226516.

4. Dorrepaal J.M. An exact solution of the Navier-Stokes equation which describes nonorthogonal stagnation-point flow in two dimensions. Journal of Fluid Mechanics, 1986, vol. 163, no.

pp. 141-147. DOI: $10.1017 / \mathrm{s} 0022112086002240$.

5. Stuart J.T. The viscous flow near a stagnation point when the external flow has uniform vorticity. Journal of the Aerospace Sciences, 1959, vol. 26, no. 2, pp. 124-125. DOI: 10.2514/8.7963.

6. Riesco-Chueca P., De la Mora J.F. Brownian motion far from equilibrium: a hypersonic approach. Journal of Fluid Mechanics, 1990, vol. 214, pp. 639-663. DOI: 10.1017/S0022112090000301.

7. Hiemenz K. Die Grenzschicht an einem in den gleichförmigen Flüssigkeitsstrom eingetauchten geraden Kreiszylinder. Dingler's Politech. J., 1911, vol. 326, pp. 321-324.

8. Ekman V.W. On the Influence of the Earth's Rotation on Ocean-Currents. Ark. Mat. Astron. Fys., 1905, vol. 2, no. 11, pp. 1-52.

9. Aristov S.N., Knyazev D.V., Polyanin A.D. Exact solutions of the Navier-Stokes equations with the linear dependence of velocity components on two space variables. Theoretical Foundations of Chemical Engineering, 2009, vol. 43, iss. 5, pp. 642-661. DOI: 10.1134/S0040579509050066.

10. Aristov S.N., Prosviryakov E.Yu. Nonuniform convective Couette flow. Fluid Dynamics, 2016, vol. 51, no. 5, pp. 581-587. DOI: 10.1134/S001546281605001X. 
11. Gorshkov A.V., Prosviryakov E.Yu. Layered B'enard-Marangoni convection during heat transfer according to the Newton's law of cooling. Kompyuternye issledovaniya $i$ modelirovanie, 2016, vol. 8, no. 6, pp. 927-940. (In Russian).

12. Gorshkov A.V., Prosviryakov E.Yu. Complex stationary convection with third-kind boundary conditions at the boundaries of a fluid layer. Diagnostics, Resource and Mechanics of Materials and Structures, 2016, iss. 2, pp. 34-47. DOI: 10.17804/2410-9908.2016.2.034-047. Available at: http://dream-journal.org/issues/2016-2/2016-2_81.html (accessed: 01.10.2017).

13. Gorshkov A.V., Prosviryakov E.Yu. Analytic solutions of stationary complex convection describing a shear stress field of different signs. Trudy Inst. Mat. i Mekh. UrO RAN, 2017, vol. 23, no. 2, pp. 32-41. DOI: 10.21538/0134-4889-2017-23-2-32-41. (In Russian).

14. Aristov S.N., Prosviryakov E.Yu., Spevak L.F. Nonstationary laminar thermal and solutal Marangoni convection of a viscous fluid. Vychislitelnaya mekhanika sploshnykh sred, 2015, vol. 8, no. 4, pp. 445-456. (In Russian).

15. Burmasheva N.V., Prosviryakov E.Yu. A large-scale layered stationary convection of an incompressible viscous fluid under the action of shear stresses at the upper boundary. Velocity field investigation. Vestn. Samar. Vestn. Samar. Gos. Tekhn. Univ., Ser. Fiz.-Mat. Nauki, 2017, vol. 21, no. 1, pp. 180-196. DOI: 10.14498/vsgtu1527. (In Russian).

16. Burmasheva N.V., Prosviryakov E.Yu. Exact solutions for layered large-scale convection induced by tangential stresses specified on the free boundary of a fluid layer. IOP Conference. Series: Materials Science and Engineering, 2017, vol. 208, conf. 1. DOI: 10.1088/1757-899X/208/1/012010. 17. Ostroumov G.A. Free convection under the condition of the internal problem. Washington, NACA Technical Memorandum 1407, National Advisory Committee for Aeronautics, 1958.

18. Birikh R. V. Thermocapillary convection in a horizontal layer of liquid. J. Appl. Mech. Tech. Phys., 1966, vol. 7, no. 3, pp. 43-44.

19. Sidorof A.F. On two classes of solutions of the equations of fluid and gas mechanics and their relation to the theory of traveling waves. Prikl. mech. $i$ tekhnich. fizika, 1989, no. 2, pp. 34-40. (In Russian).

20. Aristov S.N., Prosviryakov E.Yu. A new class of exact solutions for three-dimensional thermal diffusion equations. Theor. Found. Chem. Eng., 2016, vol. 50, no. 3, pp. 286-293. DOI: $10.1134 /$ S0040579516030027.

21. Aristov S.N., Frik P.G. Nonlinear effects of the Ekman layer on the dynamics of largescale eddies in shallow water. J. Appl. Mech. Tech. Phys., 1991, vol. 32, no. 2, pp. 189-194.

22. Ingel L.Kh., Aristov S.N. The class of exact solutions of nonlinear problems on thermal circulation associated with volumetric heat release in the atmosphere. Tr. In-ta Eksperim. Meteorol., 1996, no. 27 (162), pp. 142-157. (In Russian).

23. Aristov S.N., Shvarts K.G. Vikhrevye Techeniya Advektivnoy Prirody vo Vrashchayushchemsya Sloe Zhidkosti [Vortical Flows of the Advective Nature in a Rotating Fluid Layer]. Perm, Perm State Univ. Publ., 2006, 155 pp. (In Russian).

24. Aristov S.N., Shvarts K.G. Vikhrevye Techeniya v Tonkikh Sloyakh Zhidkosti [Vortical Flows in Thin Fluid Layers]. Kirov, Vyatka State Univ. Publ., 2011, 207 pp. (In Russian).

25. Aristov S.N., Shvarts K.G. Advective flow in a rotating liquid film. J. Appl. Mech. Tech. Phys., 2016, vol. 57, no. 1, pp. 188-194. DOI: 10.1134/S0021894416010211.

26. Aristov S.N., Prosviryakov E.Yu. On laminar flows of planar free convection. Nelin. Dinam., 2013, vol. 9, no. 4, pp. 651-657. DOI: 10.20537/nd1304004. (In Russian).

27. Andreev V.K. Resheniya Birikha uravneniy konvektsii i nekotorye ego obobshcheniya [Birikh Solutions to Convection Equations and Some of its Extensions]. Krasnoyarsk, IBM SO RAN Publ., 2010, 68 p. (In Russian).

28. Aristov S.N., Prosviryakov E.Yu., Spevak L.F. Unsteady-state Bénard-Marangoni convection in layered viscous incompressible flows. Theor. Found. Chem. Eng., 2016, vol. 50, no. 2, pp. 132-141. 
DOI: $10.1134 / \mathrm{S} 0040579516020019$.

29. Andreev V.K., Gaponenko Ya.A., Goncharova O.N., Pukhnachev V.V. Mathematical Models of Convection. Berlin, Boston, De Gryuter Publ., 2012, 417 p.

30. Pukhnachev V.V. Group-theoretical methods in the convection problems. In: Application of Mathematics in Technical and Natural Sciences, M.D. Todorov and C.I. Christov, eds., American Institute of Physics, CP 1404, Melwille, NY, 2011, pp. 31-42.

31. Pukhnachev V.V. Non-stationary analogues of the Birikh solution. Izvestiya AltGU, 2011, no. 1-2, pp. 62-69. (In Russian).

32. Andreev V.K., Bekezhanova V.B. Stability of non-isothermal fluids (Review). J. Appl. Mech. Tech. Phys., 2013, vol. 54, no. 2, pp. 171-184. DOI: 10.1134/S0021894413020016.

33. Andreev V.K., Stepanova I.V. Unidirectional flows of binary mixtures within the framework of the Oberbeck-Boussinesq model. Fluid Dyn., 2016, vol. 51, no. 2, pp. 136-147. DOI: $10.1134 / \mathrm{S} 0015462816020022$.

34. Goncharova O.N., Kabov O.A. Gravitational-thermocapillary convection of fluid in the horizontal layer in co-current gas flow. Dokl. Phys., 2009, vol. 54, no. 5, pp. 242-247. DOI: $10.1134 / \mathrm{S} 1028335809050061$.

35. Goncharova O.N., Rezanova E.V. Example of an exact solution of the stationary problem of two-layer flows with evaporation at the interface. J. Appl. Mech. Tech. Phys., 2014, vol. 55, no. 2, pp. 247-257. DOI: 10.1134/S0021894414020072.

36. Birikh R.V., Pukhnachev V.V. An axial convective flow in a rotating tube with a longitudinal temperature gradient. Dokl. Phys., 2011, vol. 56, no. 1, pp. 47-52. DOI: 10.1134/S1028335811010095.

37. Birikh R.V., Pukhnachev V.V., Frolovskaya O.A. Convective flow in a horizontal channel with non-Newtonian surface rheology under time-dependent longitudinal temperature gradient. Fluid Dyn., 2015, vol. 50, no. 1, pp. 173-179. DOI: 10.1134/S0015462815010172.

38. Ryzhkov I.I. Termodiffuziya $v$ Smesyakh: Uravneniya, Simmetrii, Resheniya $i$ Ikh Ustoychivost [Thermodiffusion in Mixtures: Equations, Symmetries, Solutions and their Stability]. Novosibirsk, SB RAS Publ., 2013, 200 p. (In Russian). 
Подана в журнал: 08.08.2017

УДК 539

DOI: $10.17804 / 2410-9908.2017 .4 .016-031$

\title{
ТОЧНЫЕ РЕШЕНИЯ ДЛЯ ЕСТЕСТВЕННОЙ КОНВЕКЦИИ СЛОИСТЫХ ТЕЧЕНИЙ ВЯЗКОЙ НЕСЖИМАЕМОЙ ЖИДКОСТИ ПРИ ЗАДАНИИ ТАНГЕНЦИАЛЬНЫХ СИЛ И ЛИНЕЙНОГО РАСПРЕДЕЛЕНИЯ ТЕМПЕРАТУРЫ НА ГРАНИЦАХ СЛОЯ
}

\author{
Н. В. Бурмашева ${ }^{1,2^{*}}$, Е. Ю. Просвиряков ${ }^{1}$ \\ ${ }^{1}$ Институт машиноведения УрО РАН, \\ ул. Комсомольская, 34, Екатеринбург, Российская Федерация \\ ${ }^{2}$ Уральский федеральный университет им. Б.Н. Ельцина, \\ ул. Мира, 19, Екатеринбург, Российская Федерациия \\ *Ответственный автор. Электронная почта: nat_burm@mail.ru \\ Адрес для переписки: ул. Комсомольская, 34, Екатеринбург, Российская Федерация \\ Тел.:+7 (343) 375-35-76; факс:374-53-30
}

В статье рассмотрено новое точное решение уравнений Обербека-Буссинеска для конвективного течения вязкой несжимаемой жидкости. Слоистые течения вязкой несжимаемой жидкости исследуются в рамках класса точных решений Сидорова-Линя, обобщающего семейство решений Остроумова-Бириха. Использование точного решения позволяет разрешить переопределенную систему уравнений движения жидкости. Нагрев жидкости осуществляется посредством задания источника тепла на обеих границах. Размерность изучаемой краевой задачи не может быть понижена преобразованием поворота. Полученное точное решение описывает противотечения в жидкости. При течении жидкости возникает термоклин и пограничный слой вблизи одной из границ слоя.

Ключевые слова: слоистое течение, точное решение, противотечения.

\section{1. Введение}

Изучение конвективных движений вязкой жидкости является одной из распространенных задач в самых разных теоретических и прикладных научных дисциплинах. В условиях нормальной гравитации для описания конвективных течений жидкости используется подход Обербека [1]. Интерес к нахождению точных решений обусловлен нелинейностью системы Обербека-Буссинеска и изучением физических механизмов теплового движения жидкости, которые часто обладают весьма неожиданными свойствами относительно движений жидкости при постоянной температуре $[1,2]$.

Особо интересным оказывается вопрос о наличии в слое жидкости застойных точек (точек покоя). Если решения уравнений Навье-Стокса и их модификации являются непрерывными функциями координат, то рядом с такими точками, как правило, образуются застойные зоны, т. е. области с обратным (возвратным) течением [3-6].

Первым изотермическим точным решением, посвященным исследованию застойных точек, является течение Хименца [7]. Исследование противотетечений в океане было начато Экманом [8]. Известно, что течение Экмана является сдвиговым, у которого вертикальная скорость равна нулю (слоистым) [9]. Влияние температурной стратификации может существенно изменить структуру поля скоростей. В этом случае в жидкости могут появиться дополнительные точки покоя в сравнении с изотермическими течениями. Соответствующие условия для различных видов краевых задач конвекции представлены в [10-16]. 
Исследование конвективных течений в аналитической форме осуществляется посредством построения и анализа классов точных решений уравнений Обербека-Буссинеска. В [17-31] построены широкие классы точных решений для описания жидкостей при вращении. В статьях [32-38] приведены точные решения для конвективных термокапиллярных течений, которые являются обобщениями течения Остроумова-Бириха $[17,18]$. Примеры точных решений, описывающих изотермические и конвективные противотечения в слоистых течениях для вертикально завихренной жидкости, представлены в [10].

В работе предложено новое точное решение, описывающее движение вязкой несжимаемой жидкости под действием заданных на верхней границе тангенциальных сил, которые не являются в общем случае результатом действия капиллярного эффекта. Характерной особенностью полученного решения является трехмерность полей давления и температуры по координатам. Кроме того, данное решение при определенных значениях параметров, задаваемых на границах слоя жидкости, допускает появление расслоений физических полей, причем точек расслоения может быть несколько.

\section{2. Постановка задачи и методы решения}

Для описания конвективных движений в слоистых крупномасштабных течениях вязкой несжимаемой жидкости в стационарном случае, как правило, используется система уравнений тепловой конвекции в приближении Буссинеска $[1,2]$. С математической точки зрения эта система состоит из уравнения Навье-Стокса

$$
(\boldsymbol{V} \cdot \nabla) \boldsymbol{V}=-\nabla P+v \Delta \boldsymbol{V}+g \beta T \boldsymbol{k},
$$

уравнения теплопроводности

$$
\boldsymbol{V} \cdot \nabla T=\chi \Delta T
$$

и уравнения несжимаемости

$$
\nabla \cdot \boldsymbol{V}=0
$$

В системе (1)-(3) используются стандартные обозначения: $\boldsymbol{V}(x, y, z)=\left(V_{x}, V_{y}, V_{z}\right)-$ вектор скорости, причем $V_{z} \equiv 0$, так как рассматривается слоистое течение; $P$ - отклонение давления от гидростатического, деленное на постоянную среднюю плотность с жидкости, линейно зависящую от температуры; $T$ - отклонение от средней температуры; $v, x$ - коэффициенты кинематической вязкости и температуропроводности жидкости соответственно; $\boldsymbol{k}$ - орт оси $O z$,направленной вертикально вверх; $\nabla=\left(\frac{\partial}{\partial x}, \frac{\partial}{\partial y}, \frac{\partial}{\partial z}\right), \Delta=\frac{\partial^{2}}{\partial x^{2}}+\frac{\partial^{2}}{\partial y^{2}}+\frac{\partial^{2}}{\partial z^{2}}-$ оператор Гамильтона и двумерный оператор Лапласа соответственно.

Полученная система (1)-(3) является нелинейной (за счет наличия конвективных членов в уравнении теплопроводности) и переопределенной (5 скалярных уравнений относительно четырех неизвестных - компонент скорости $V_{x}, V_{y}$ и физических полей $P$ и $T$ ). Чтобы избавиться от переопределенности, будем искать решение в классе, предложенном в [29]:

$$
\begin{gathered}
V_{x}=u(z) ; \quad V_{y}=v(z) ; \\
T=T_{0}(z)+T_{1}(z) x+T_{2}(z) y ; \quad P=P_{0}(z)+P_{1}(z) x+P_{2}(z) y .
\end{gathered}
$$

Использование класса (4) приводит к тому, что уравнение неразрывности удовлетворяется тождественно: 


$$
\frac{\partial V_{x}}{\partial x}+\frac{\partial V_{y}}{\partial y}+\frac{\partial V_{z}}{\partial z}=\frac{\partial u(z)}{\partial x}+\frac{\partial v(z)}{\partial y} \equiv 0
$$

Подставляя разложения (4)-(5) в уравнения Навье-Стокса (1), согласно принципу неопределенных коэффициентов получаем:

$$
\begin{aligned}
& P_{1}=v u^{\prime \prime} ; P_{2}=v v^{\prime \prime} ; \\
& \frac{\partial P_{i}}{\partial z}=g \beta T_{i} ; i=0,1,2 .
\end{aligned}
$$

Аналогичным образом из уравнения теплопроводности получаем еще три уравнения:

$$
u T_{1}+v T_{2}=\chi T_{0}^{\prime \prime} ; T_{1}^{\prime \prime}=T_{2}^{\prime \prime}=0 .
$$

Таким образом, окончательно получаем систему уравнений:

$$
\begin{gathered}
\frac{\partial^{2} T_{1}}{\partial z^{2}}=0 ; \quad \frac{\partial^{2} T_{2}}{\partial z^{2}}=0 ; \\
\frac{\partial P_{1}}{\partial z}=g \beta T_{1} ; \frac{\partial P_{2}}{\partial z}=g \beta T_{2} ; \\
v \frac{\partial^{2} u}{\partial z^{2}}=P_{1} ; \quad v \frac{\partial^{2} v}{\partial z^{2}}=P_{2} ; \\
\chi \frac{\partial^{2} T_{0}}{\partial z^{2}}=u T_{1}+v T_{2} ; \\
\frac{\partial P_{0}}{\partial z}=g \beta T_{0} .
\end{gathered}
$$

Полученная система дифференциальных уравнений (6)-(10) является системой тринадцатого порядка, следовательно, необходимо задать на границах слоя тринадцать условий, которые будут использованы для определения констант, возникающих при интегрировании этой системы.

Нижняя граница жидкости $(z=0)$ полагается абсолютно твердой и неподвижной, на ней выполнено условие прилипания $\left(V_{x}(0)=V_{y}(0)=0\right)$ и задано температурное поле, которое в силу структуры выражений (5) записывается в виде

$$
T(0)=A x+B y .
$$

Верхняя граница $(z=h)$ полагается свободной и недеформируемой при движении жидкости. Температура задается функцией

$$
T(h)=\theta+C x+D y .
$$

Давление $P(h)$ на верхней границе полагается равным $S$. Кроме того, на свободной поверхности заданы напряжения:

$$
\eta \frac{\partial u}{\partial z}=\xi_{1} ; \eta \frac{\partial v}{\partial z}=\xi_{2} .
$$

Таким образом, окончательно приходим к следующей системе граничных условий:

$$
u(0)=v(0)=0 ;
$$


http://dream-journal.orn

$$
\begin{array}{ccc}
T_{0}(0)=0 ; & T_{1}(0)=A ; & T_{2}(0)=B ; \\
T_{0}(0)=\theta ; & T_{1}(0)=C ; & T_{2}(0)=D ; \\
P_{0}(h)=S ; & P_{1}(h)=0 ; & P_{2}(h)=0 ; \\
\eta \frac{\partial u}{\partial z}(h)=\xi_{1} ; & \eta \frac{\partial v}{\partial z}(h)=\xi_{2} .
\end{array}
$$

Далее будем рассматривать частный случай задания температурного возмущения только на нижней границе $(C=D=0)$. Кроме того, будем полагать $S=0$, т. е. отсчет приведенного давления будет вестись от уровня, задаваемого на верхней границе слоя.

\section{3. Результаты и обсуждение}

\section{1 Получение решения краевой задачи}

Интегрируя последовательно уравнения системы (6)-(10) в силу приведенных граничных условий, легко получаем точное решение описанной краевой задачи:

$$
\begin{gathered}
u(z)=\frac{z\left(24 h \xi_{1} v+6 B \beta \eta g h^{2} z-8 B \beta \eta g h z^{2}+3 B \beta \eta g z^{3}\right)}{24 \eta h v} ; \\
v(z)=\frac{z\left(24 h \xi_{1} v+6 A \beta \eta g h^{2} z-8 A \beta \eta g h z^{2}+3 A \beta \eta g z^{3}\right)}{24 \eta h v} ; \\
P_{0}(z)=\frac{1}{20160 \chi \eta h^{2} v} \\
\left.+A B \eta \beta g\left(35 h^{8}-80 h^{6} z^{2}+168 h^{3} z^{5}-196 h^{2} z^{6}+88 h z^{7}-15 z^{8}\right)\right\} ; \\
P_{1}(z)=-\frac{A \beta g(h-z)^{2}}{2 h} ; \\
P_{2}(z)=-\frac{B \beta g(h-z)^{2}}{2 h} ; \\
T_{0}(z)=-\frac{B}{2520 \chi \eta h^{2} v}\left\{210 h v\left[-12 \chi \eta \theta+\left(A \xi_{1}+B \xi_{2}\right)\left(h^{3}-2 h z^{2}+z^{3}\right)\right]+\right. \\
\left.+A B \eta \beta g\left(20 h^{6}-105 h^{3} z^{3}+147 h^{2} z^{4}-77 h z^{5}+15 z^{6}\right)\right\} ; \\
T_{1}(z)=A-\frac{A z}{h} ; \\
T_{2}(z)=B-\frac{B z}{h} .
\end{gathered}
$$

Поставим далее задачу исследования условий существования застойных точек и возникновения противотоков в полях давления и температуры (аналогичное исследование для поля скоростей было проведено авторами ранее, в частности в $[15,16])$. Отметим только, что 
случай $A=B=0$ не будем рассматривать за его тривиальностью. Действительно, если последнее двойное равенство будет выполнено, то получим

$$
T_{1}=T_{2}=P_{1}=P_{2} \equiv 0 ; \quad P_{0}(z)=\frac{\beta g \theta\left(z^{2}-h^{2}\right)}{2 h} ; \quad T_{0}(z)=\frac{z \theta}{h},
$$

и очевидно, что расслоений в слое $[0, h]$ не возникает.

\section{4. Нормировка решения}

Для удобства дальнейшего анализа пронормируем полученные решения для поля температуры и давления, приведя их к безразмерному виду. Пусть (без ограничения общности) $A \neq 0$. Введем новые параметры системы: $1=\frac{A}{A} ; \Delta^{*}=\frac{B}{A} ; \delta=\frac{h}{l}$, где $h, l$ - соответственно вертикальный и горизонтальный характерные размеры слоя. Далее перейдем к безразмерным координатам $\bar{x}=\frac{x}{l} ; \bar{y}=\frac{y}{l} ; \bar{z}=\frac{z}{h}$, изменяющимся в диапазоне $[0,1]$.

Функцию температуры нормируем на $A l$ :

$$
\begin{gathered}
\bar{T}=\frac{T}{A l}=\frac{T_{0}}{A l}+\frac{T_{1}}{A l} \cdot \frac{x}{l} l+\frac{T_{2}}{A l} \cdot \frac{y}{l} l=\bar{T}_{0}+\bar{T}_{1} \bar{x}+\bar{T}_{2} \bar{y} ; \\
\bar{T}_{1}=1-\bar{z}, \bar{T}_{2}=\Delta^{*}(1-\bar{z})=\Delta^{*} \bar{T}_{1} ; \\
\bar{T}_{0}=\frac{T_{0}}{A l}=\frac{1}{A l} \frac{z h v \chi \eta \theta}{\chi \eta h^{2} v}-\frac{z h v\left(A \xi_{1}+B \xi_{2}\right)\left(h^{3}-2 h z^{2}+z^{3}\right)}{A l \cdot 12 \chi \eta h^{2} v}- \\
-\frac{A B z \eta \beta g\left(20 h^{6}-105 h^{3} z^{3}+147 h^{2} z^{4}-77 h z^{5}+15 z^{6}\right)}{A l \cdot 2520 \chi \eta h^{2} v}= \\
=\frac{\theta}{A l} \frac{z}{h}-\frac{z}{h} \frac{\left(\xi_{1}+\Delta^{*} \xi_{2}\right)}{12 \chi \eta} h^{2} \frac{h}{l} \frac{\left(h^{3}-2 h z^{2}+z^{3}\right)}{h^{3}}- \\
-\frac{z}{h} \frac{B \beta g}{2520 \chi h v} \frac{\left(20 h^{6}-105 h^{3} z^{3}+147 h^{2} z^{4}-77 h z^{5}+15 z^{6}\right)}{h^{6}} \frac{h^{6}}{l}= \\
=\frac{\theta \bar{z}}{A l}-\bar{z} \frac{\left(\xi_{1}+\Delta^{*} \xi_{2}\right)}{12 \chi \eta} h^{2} \delta\left(1-2 \bar{z}^{2}+\bar{z}^{3}\right)-\bar{z} \frac{\delta \cdot P e}{2520}\left(20-105 \bar{z}^{3}+147 \bar{z}^{4}-77 \bar{z}^{5}+15 \bar{z}^{6}\right),
\end{gathered}
$$

где $P e=P r \cdot G r=\frac{v}{\chi} \cdot \frac{B h \cdot g \beta h^{3}}{v^{2}}-$ число Пекле. Можно показать, что величины $\gamma=\frac{\theta}{A l}$; $\alpha=\frac{\left(\xi_{1}+\Delta^{*} \xi_{2}\right)}{12 \chi \eta} h^{2} \delta$ также являются безразмерными. Окончательно получаем:

$$
\begin{aligned}
\bar{T}_{0} & =\gamma \bar{z}-\bar{z} \alpha\left(1-2 \bar{z}^{2}+\bar{z}^{3}\right)-\bar{z} \frac{\delta \cdot P e}{2520}\left(20-105 \bar{z}^{3}+147 \bar{z}^{4}-77 \bar{z}^{5}+15 \bar{z}^{6}\right)= \\
& =\bar{z}\left[\gamma-\alpha\left(1-2 \bar{z}^{2}+\bar{z}^{3}\right)-\frac{\delta \cdot P e}{2520}\left(20-105 \bar{z}^{3}+147 \bar{z}^{4}-77 \bar{z}^{5}+15 \bar{z}^{6}\right)\right]
\end{aligned}
$$


Аналогично обезразмериваем давление, разделив все члены на $g \beta A l^{2}$ :

$$
\begin{aligned}
& \bar{P}=\frac{P}{g \beta A l^{2}}=\frac{P_{0}}{g \beta A l^{2}}+\frac{P_{1}}{g \beta A l} \cdot \frac{x}{l}+\frac{P_{2}}{g \beta A l} \cdot \frac{y}{l}=\bar{P}_{0}+\bar{P}_{1} \bar{x}+\bar{P}_{2} \bar{y} ; \\
& \bar{P}_{1}=\frac{P_{1}}{g \beta A l}=-(1-\bar{z})^{2} \cdot \frac{\delta}{2} ; \quad \bar{P}_{2}=\frac{P_{2}}{g \beta A l}=-\Delta^{*}(1-\bar{z})^{2} \cdot \frac{\delta}{2}=\Delta^{*} \bar{P}_{1} \text {; } \\
& \bar{P}_{0}=\frac{1}{g \beta A l^{2}} \cdot \frac{168 * 60 h v \chi \eta \beta g \theta\left(z^{2}-h^{2}\right)}{20160 \chi \eta h^{2} v} \cdot \frac{h^{2}}{h^{2}}+ \\
& +\frac{168 h v \beta g\left(A \xi_{1}+B \xi_{2}\right)\left(2 h^{5}-5 h^{3} z^{2}+5 h z^{4}-2 z^{5}\right)}{g \beta A l^{2} \cdot 20160 \chi \eta h^{2} v} \cdot \frac{h^{5}}{h^{5}}+ \\
& +\frac{A B \eta \beta^{2} g^{2}\left(35 h^{8}-80 h^{6} z^{2}+168 h^{3} z^{5}-196 h^{2} z^{6}+88 h z^{7}-15 z^{8}\right)}{g \beta A l^{2} \cdot 20160 \chi \eta h^{2} v} \cdot \frac{h^{8}}{h^{8}}= \\
& =\frac{\theta\left(\bar{z}^{2}-1\right)}{2 A l} \cdot \frac{h}{l}+\frac{\left(\xi_{1}+\Delta^{*} \xi_{2}\right)}{12 \chi \eta} \cdot \frac{1}{l^{2}} \cdot \frac{h}{h^{2}}\left(2-5 \bar{z}^{2}+5 \bar{z}^{4}-2 \bar{z}^{5}\right) h^{5}+ \\
& +\frac{1}{20160} \cdot \frac{B \beta g h^{4}}{v^{2}} \cdot \frac{v}{\chi} \delta^{2}\left(35-80 \bar{z}^{2}+168 \bar{z}^{5}-196 \bar{z}^{6}+88 \bar{z}^{7}-15 \bar{Z}^{8}\right)= \\
& =\gamma\left(\bar{z}^{2}-1\right) \cdot \frac{\delta}{2}+\frac{\alpha \delta^{2}}{10}\left(2-5 \bar{z}^{2}+5 \bar{z}^{4}-2 \bar{z}^{5}\right)+ \\
& +\frac{P e \cdot \delta^{2}}{20160}\left(35-80 \bar{z}^{2}+168 \bar{z}^{5}-196 \bar{z}^{6}+88 \bar{z}^{7}-15 \bar{z}^{8}\right) .
\end{aligned}
$$

\section{5. Исследование решения}

Для дальнейшего удобства всюду далее опустим «крышечки» у нормированных функций и приведенных переменных. Отметим, что функции $T(z) ; P(z)$ линейны по $x, y$ и значительно нелинейны по $z$. Исследовать эти функции на экстремум в таком виде неудобно, так как они описывают поверхности в четырехмерном пространстве координат, однако в данном случае можно снизить размерность, введя замену $t=x+\Delta^{*} y \in[0,1+$ $\left.+\Delta^{*}\right]$. Можно показать, что точка $\left(x_{*}, y_{*}, z_{*}\right)$ является экстремумом функции $T(z)$ (или $P(z))$ тогда и только тогда, когда экстремумом этой функции является точка $\left(t_{*}, z_{*}\right)$, где $t=x_{*}+\Delta^{*} y_{*}$.

Необходимым условием существования экстремума является обращение в ноль частных производных исследуемой функции. Найдем эти производные на примере функции температуры $T(z)$ :

$$
\frac{\partial T}{\partial t}=1-z=0, \text { т. е. } z_{*}=1
$$




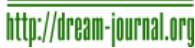

$$
\begin{aligned}
& \frac{\partial T}{\partial z}=\gamma+\alpha\left(-1+3 z^{2}-4 z^{3}\right)-\frac{P e \cdot \delta}{2520}\left(20-420 z^{3}+147 * 5 z^{4}-77 * 6 z^{5}+15 * 7 z^{6}\right)-t=0 ; \\
& \frac{\partial T}{\partial z_{z_{*}=1}}=\gamma-2 \alpha+\frac{3 P e \cdot \delta}{280}-t_{*}=0 ; \quad t_{*}=\gamma+\frac{3 P e \cdot \delta}{280}-2 \alpha .
\end{aligned}
$$

Вопрос о том, попадает ли точка $t_{*}$ в интервал $\left[0,1+\Delta^{*}\right]$,оставим пока открытым.

Достаточное условие существования экстремума у функции $f(t, z)$ двух переменных заключается в определении знака выражения

$$
\frac{\partial^{2} f}{\partial t^{2}} \cdot \frac{\partial^{2} f}{\partial z^{2}}-\left(\frac{\partial^{2} f}{\partial t \partial z}\right)^{2}
$$

в точке, подозрительной на экстремум. В случае функции температуры имеем:

$$
\frac{\partial^{2} T}{\partial t^{2}} \cdot \frac{\partial^{2} T}{\partial z^{2}}-\left(\frac{\partial^{2} T}{\partial t \partial z}\right)^{2}=0 \cdot \frac{\partial^{2} T}{\partial z^{2}}-\left(\frac{\partial^{2} T}{\partial t \partial z}\right)^{2}=-\left(\frac{\partial^{2} T}{\partial t \partial z}\right)^{2}=-1<0
$$

т. е., экстремума у функции $T$ нет. Найденная выше точка $\left(t_{*}, z_{*}\right)$ является седловой. Аналогично доказываем, что экстремума нет и у функции давления $P$. Однако отсутствие экстремума у этих функций не гарантирует отсутствие застойных точек, определяющих положение расслоений полей.

Как отмечалось выше, функции $T(t, z)$ и $P(t, z)$ существенно нелинейны по $z$, а значит, их поведение зависит от значений параметров, задаваемых на границе. На рис. 1 представлены поверхности, задаваемые функцией температуры $T(t, z)$, в трехмерном пространстве $(t, z, T(t, z))$ для трех различных наборов значений параметров, определяющих граничные условия.

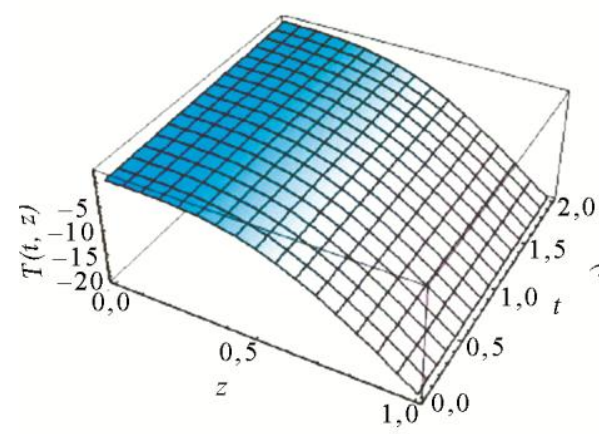

$a$

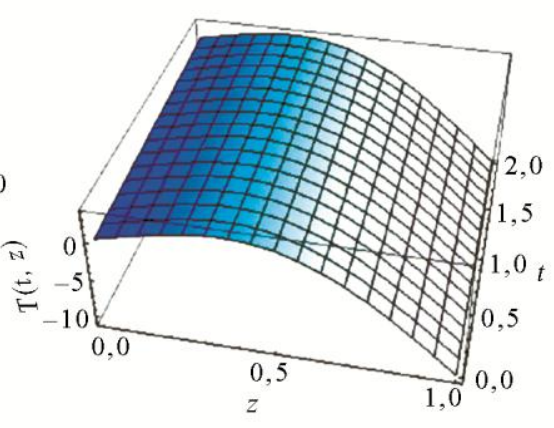

$\sigma$

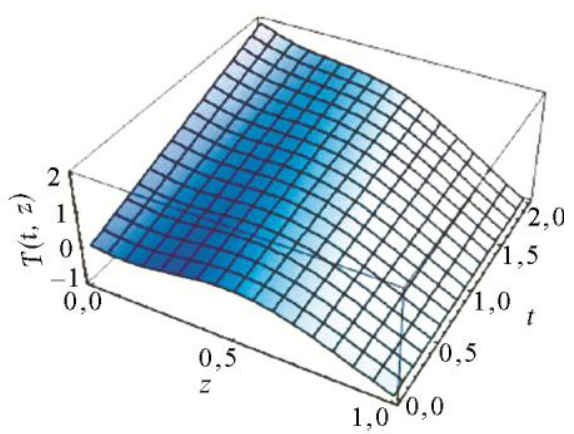

B

Рис. 1. Поведение функции $T(t, z)$ в зависимости от значений параметров системы:

$$
\begin{aligned}
a-\text { при } \frac{\left(A \xi_{1}+B \xi_{2}\right) h^{3}}{12 A \chi \eta l} & =-1 ; \frac{\theta}{A l}=-19 ; \sigma-\text { при } \frac{\left(A \xi_{1}+B \xi_{2}\right) h^{3}}{12 A \chi \eta l}=-1 ; \frac{\theta}{A l}=-9 ; \\
\text { в } & - \text { при } \frac{\left(A \xi_{1}+B \xi_{2}\right) h^{3}}{12 A \chi \eta l}=-20 ; \frac{\theta}{A l}=-1
\end{aligned}
$$


Аналогично на рис. 2 приведена поверхность, задаваемая функцией давления $P(t, z)$.

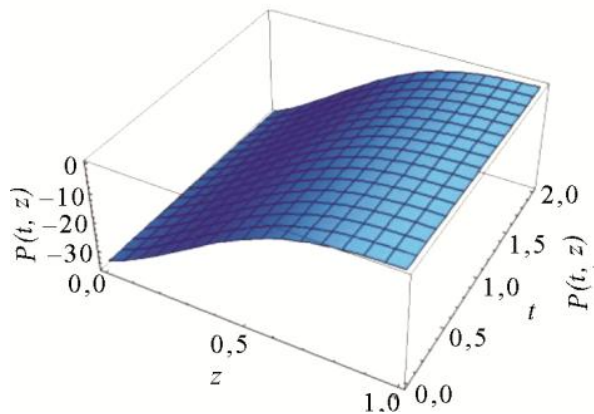

$a$

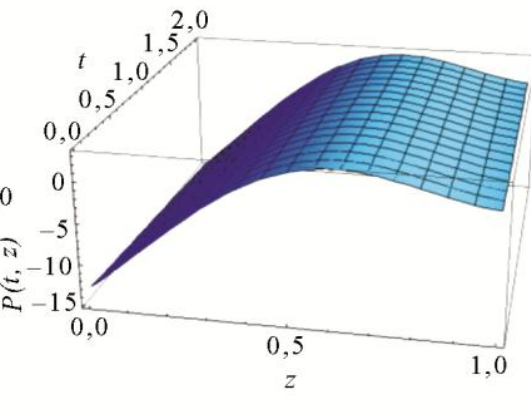

$\sigma$

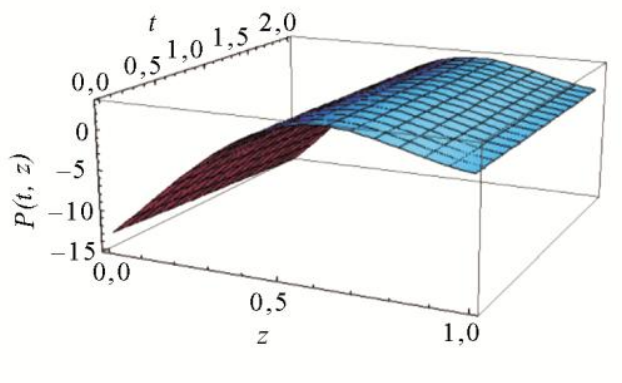

B

Рис. 2. Поведение функции $P(t, z)$ в зависимости от значений параметров системы:

$$
\begin{aligned}
& a-\text { при } \frac{\left(A \xi_{1}+B \xi_{2}\right) h^{5}}{120 A \chi \eta l^{3}}=1 ; \frac{\theta}{2 A l^{2}}=1 ; \sigma-п р и \frac{\left(A \xi_{1}+B \xi_{2}\right) h^{5}}{120 A \chi \eta l^{3}}=10 ; \frac{\theta}{2 A l^{2}}=-2 ; \\
& в-\text { при } \frac{\left(A \xi_{1}+B \xi_{2}\right) h^{5}}{120 A \chi \eta l^{3}}=10 ; \frac{\theta}{2 A l^{2}}=2,5
\end{aligned}
$$

Кроме того, возможны такие сочетания параметров системы, что внутри изучаемого слоя можно выделить подслои так, что хотя бы на границе каждого такого подслоя температура $T(z)$ (или, соответственно, давление $P(t, z))$ становится нулевой. Границы $z_{i}$ этих подслоев являются нулями функции $T_{0}$, если исследуется поле температуры, и функции $P_{0}$, если речь идет об исследовании давления.

Начнем с функции температуры $T_{0}$. Очевидно, что $z_{0}=0$ есть нуль функции $T_{0}$, однако он совпадает с одним из концов исследуемого отрезка $[0,1]$, поэтому не определяет подслой с противотечением.

Введем функцию $f(z)$ следующим образом:

$$
T_{0}=z \cdot\left(-\frac{\delta \cdot P e}{2520}\right) \cdot f(z)
$$

где $f(z)=15 z^{6}-77 z^{5}+147 z^{4}+(a-105) z^{3}-2 a z^{2}+b ; a=\frac{2520 \alpha}{\delta \cdot P e}, b=20+\frac{2520(\alpha-\gamma)}{\delta \cdot P e}$.

Функция $f(z)$ является полиномом шестой степени относительно $z$. Следовательно, у нее не более шести корней, но необязательно все из них будут принадлежать отрезку $[0,1]$, поэтому не могут определять точку расслоения. Согласно теореме Декарта число положительных корней многочлена с вещественными коэффициентами равно количеству перемен знаков в ряду его коэффициентов или на четное число меньше этого количества.

\begin{tabular}{|c|c|c|c|c|}
\hline \multirow{2}{*}{$\begin{array}{c}\text { № } \\
\text { П.П. }\end{array}$} & \multicolumn{3}{|c|}{ Параметры } & \multirow{2}{*}{$\begin{array}{c}\text { Число смен } \\
\text { знаков }\end{array}$} \\
\hline & $a-105$ & $a$ & $b$ & \\
\hline 1 & $a-105<0$ & $a>0$ & $b \geq 0$ & 4 \\
\hline 2 & $-\gg-$ & $-\gg-$ & $b<0$ & 5 \\
\hline 3 & $-\gg-$ & $a \leq 0$ & $b>0$ & 4 \\
\hline 4 & $-\gg-$ & $-\gg-$ & $b \leq 0$ & 3 \\
\hline 5 & $a-105 \geq 0$ & $a \geq 0$ & $b \geq 0$ & 2 \\
\hline 6 & $-»-$ & $-\gg-$ & $b<0$ & 3 \\
\hline 7 & - & $a<0-$ не реализуем & - & - \\
\hline
\end{tabular}

В таблице приведено возможное количество смен знаков ряда коэффициентов функции $f(z)$ в зависимости от значений параметров $a, b$.

Burmasheva N.V. et al. Exact solutions for natural convection of layered flows

of a viscous incompressible fluid with Specified tangential forces and the linear distribution

of temperature on the layer boundaries 
Таким образом, у функции $f(z)$ может быть не более 5 положительных корней. Открытым остается вопрос о том, сколько из них будет $<1$.

Введем функции $f_{1}(z), f_{2}(z)$ следующим образом:

$$
f(z)=f_{1}(z)+a f_{2}(z)+b,
$$

где $f_{1}(z)=15 z^{6}-77 z^{5}+147 z^{4}-105 z^{3} ; f_{2}(z)=z^{3}-2 z^{2}$.

Нетрудно показать, что введенные функции $f_{1}(z), f_{2}(z)$ монотонно убывают на интервале $(0,1)$. Тогда при неотрицательном $a$ функция $f(z)$ будет монотонно убывать, а значит, будет иметь на интервале $(0,1)$ не более одного нуля (рис. 3 ). В этих случаях функция температуры $T(t, z)$ задает поверхность, вид которой приведен, соответственно, на рис. $1 a, \sigma$.

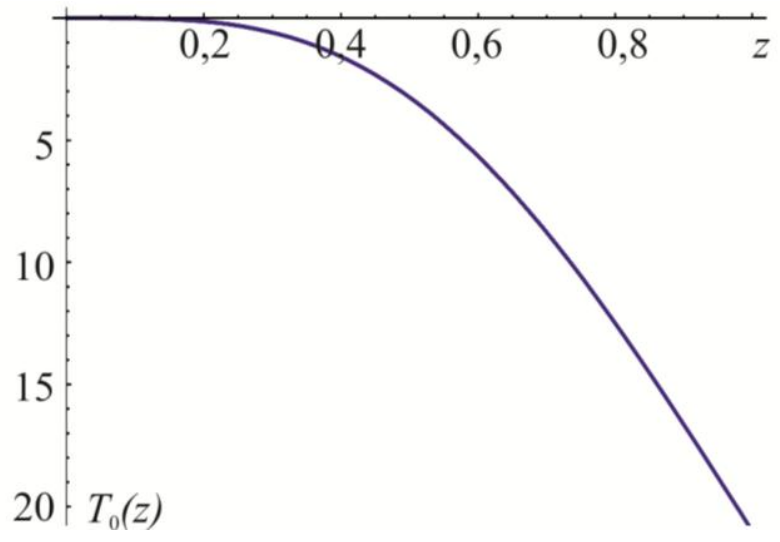

$a$

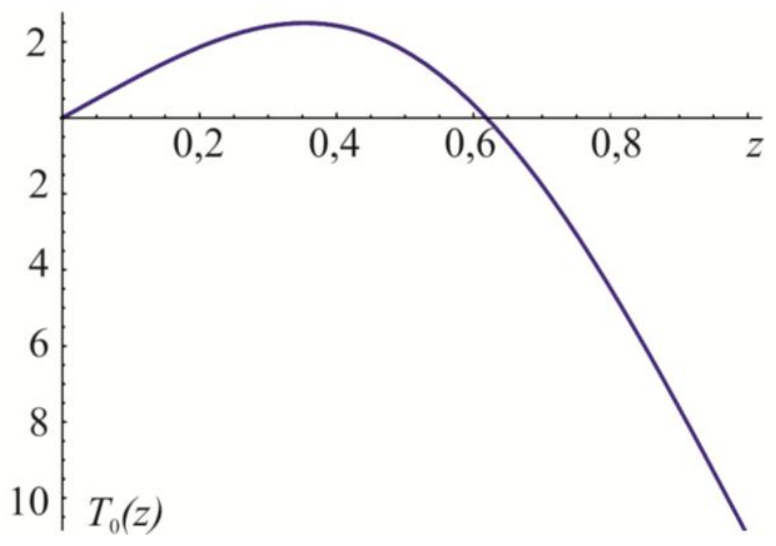

$\sigma$

Рис. 3. Поведение функции $T_{0}(z)$ : при $a=1, b=0$ (a); при $a=1, b=10$ (б)

При отрицательном $a$ функция $f(z)$ представляет собой сумму также монотонных функций, но уже разнонаправленных (убывающей и возрастающей), поэтому в данном случае функция $f(z)$ (а, следовательно, и функция $T_{0}$ ) может иметь уже большее число нолей в интервале $(0,1)$. На рис. 4 показан случай, когда функция $T_{0}$ имеет два ноля, а функция температуры $T(t, z)$ имеет вид, представленный на рис. 1 в.

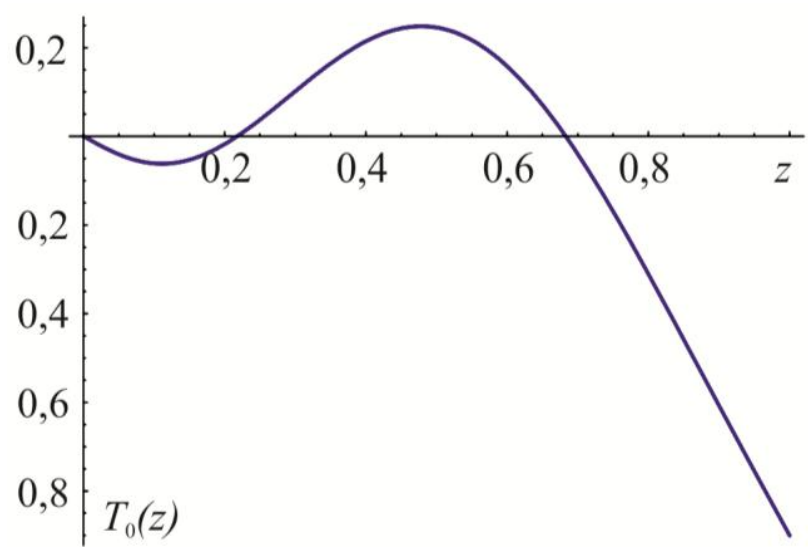

Рис. 4. Поведение функции $T_{0}(z)$ : при $a=-20, b=1$ 
Аналогичные рассуждения справедливы и при анализе функции давления $P_{0}$.

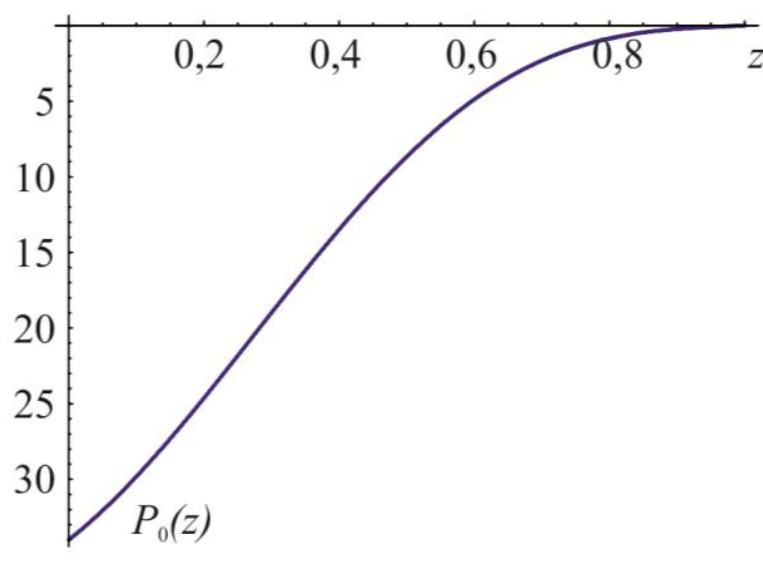

$a$

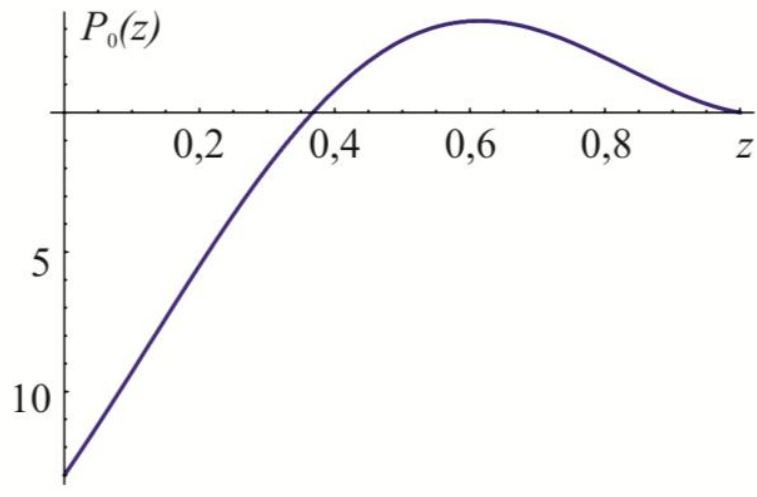

6

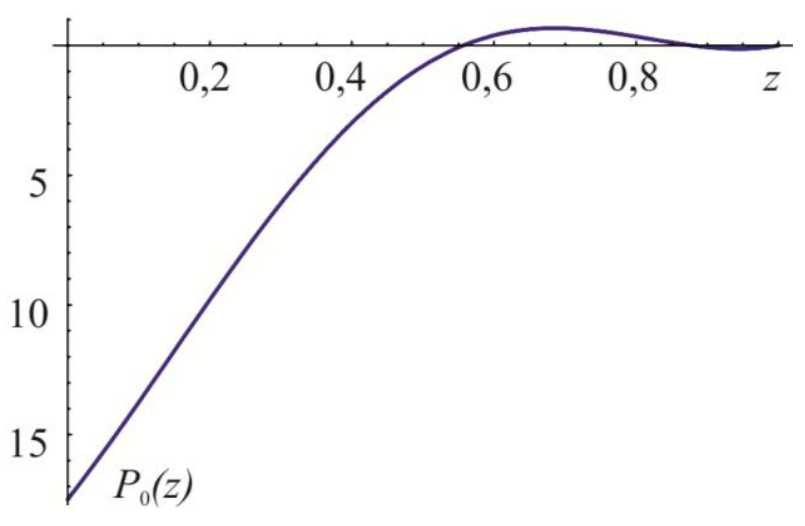

B

Рис. 5. Поведение функции $P_{0}(z): a-$ при $a=-\frac{10}{\delta^{2}} ; b=20+\frac{2}{\delta}+\frac{10}{\delta^{2}}$,

$\sigma-$ при $a=-\frac{100}{\delta^{2}} ; b=20-\frac{4}{\delta}+\frac{100}{\delta^{2}}, в-$ при $a=-\frac{100}{\delta^{2}} ; b=20+\frac{5}{\delta}+\frac{100}{\delta^{2}}$

Когда у компоненты $P_{0}(z)$ нет нулей на исследуемом интервале (рис. $5 a$ ), поле давления $P(t, z)$ определяется поверхностью, изображенной на рис. $2 a$; когда есть один нуль (рис. 5 б) - поверхностью, изображенной на рис. 2 б; наконец, когда есть, например, два нуля (рис. 5 в) - поверхностью, изображенной на рис. 2 в. Кроме того, напомним, что ввиду выбранной выше замены $t=x+\Delta^{*} y$ каждой паре точек $(t, z)$ на рис. 1 и 2 соответствует целая прямая в изначальном пространстве координат $(x, y, z)$, введенных в слое жидкости при формулировке основных соотношений модели.

\section{6. Заключение}

В настоящей работе были рассмотрены слоистые крупномасштабные течения вязкой несжимаемой жидкости в стационарном случае, индуцируемые градиентом температуры и касательными напряжениями на границе. Было получено точное решение системы уравнений тепловой конвекции в приближении Буссинеска для заданных краевых условий. Для полученного решения, описывающего изменение физических полей, было показано, что при некоторых условиях найдется толщина слоя жидкости, при которой в слое появляются точки, в которых однородные (относительно горизонтальных координат) 
члены функций температуры и давления обращаются в ноль. Кроме того, при переходе через эти точки значения температуры и давления меняют знак на противоположный. Также было показано, что если такие точки в слое жидкости есть, то они могут быть неединственные.

\section{Благодарность}

Работа выполнена при поддержке фонда содействия развитию мальх форм предприятий в научно-технической сфере (программа УМНИК).

\section{Литература}

1. Джозеф Д. Д. Устойчивость движений жидкости. - М. : Мир, 1981.

2. Gershuni G. Z., Zhukhovitskii E. M. Convective Stability of Incompressible Fluids. Israel Program for Scientific Translations. - Jerusalem : Keter Publishing House, 1976. - 330 p.

3. Shtern V. Counterflows. Paradoxical Fluid Mechanics Phenomena. - Cambridge : Cambridge University Press, 2012. - 469 p. - DOI: 10.1017/CBO9781139226516.

4. Dorrepaal J. M. An exact solution of the Navier-Stokes equation which describes nonorthogonal stagnation-point flow in two dimensions // Journal of Fluid Mechanics. - 1986. Vol. 163, no. 1. - P. 141-147. - DOI: 10.1017/s0022112086002240.

5. Stuart J. T. The viscous flow near a stagnation point when the external flow has uniform vorticity // Journal of the Aerospace Sciences. - 1959. - Vol. 26, no. 2. - P. 124-125. DOI: $10.2514 / 8.7963$.

6. Riesco-Chueca P., De la Mora J. F. Brownian motion far from equilibrium: a hypersonic approach // Journal of Fluid Mechanics. - 1990. - Vol. 214. - P. 639-663. - DOI: 10.1017/S0022112090000301.

7. Hiemenz K. Die Grenzschicht an einem in den gleichförmigen Flüssigkeitsstrom eingetauchten geraden Kreiszylinder // Dingler's Politech. J. - 1911. - Vol. 326. - P. 321-324.

8. Ekman V. W. On the Influence of the Earth's Rotation on Ocean-Currents // Ark. Mat. Astron. Fys. - 1905. - Vol. 2, no. 11. - P. 1-52.

9. Aristov S. N., Knyazev D. V., Polyanin A. D. Exact solutions of the Navier-Stokes equations with the linear dependence of velocity components on two space variables // Theoretical Foundations of Chemical Engineering. - 2009. - Vol. 43, iss 5. - P. 642-661. - DOI: 10.1134/S0040579509050066.

10. Aristov S. N., Prosviryakov E. Yu. Nonuniform convective Couette flow // Fluid Dynamics. 2016. - Vol. 51, no. 5. - P. 581-587. - DOI: 10.1134/S001546281605001X.

11. Горшков А. В., Просвиряков Е. Ю. Слоистая конвекция Бенара-Марангони при теплообмене по закону Ньютона-Рихмана // Компьютерные исследования и моделирование. 2016. - T. 8, № 6. - C. 927-940.

12. Gorshkov A. V., Prosviryakov E. Yu. Complex stationary convection with third-kind boundary conditions at the boundaries of a fluid layer // Diagnostics, Resource and Mechanics of Materials and Structures. - 2016. iss. 2. - P. 34-47. - DOI: 10.17804/2410-9908.2016.2.034-047. URL: http://dream-journal.org/issues/2016-2/2016-2_81.html.

13. Gorshkov A. V., Prosviryakov E. Yu. Analytic solutions of stationary complex convection describing a shear stress field of different signs // Trudy Inst. Mat. i Mekh. UrO RAN. - 2017. Vol. 23, no. 2. - P. 32-41. - DOI: 10.21538/0134-4889-2017-23-2-32-41.

14. Аристов С. Н., Просвиряков Е. Ю., Спевак Л. Ф. Нестационарная слоистая тепловая и концентрационная конвекция Марангони вязкой жидкости // Вычисл. мех. сплошн. сред. 2015. - Т. 8, № 4. - С. 445-456.

15. Бурмашева Н. В., Просвиряков Е. Ю. Крупномасштабная слоистая стационарная конвекция вязкой несжимаемой жидкости под действием касательных напряжений на верхней границе. Исследование поля скоростей // Вестн. Самарск. гос. техн. унив. Сер. физ.-мат. науки. 2017. - T. 21, № 1. - C. 180-196. - DOI: 10.14498/vsgtu1527. 
16. Burmasheva N. V., Prosviryakov E. Yu. Exact solutions for layered large-scale convection induced by tangential stresses specified on the free boundary of a fluid layer // IOP Conference Series: Materials Science and Engineering. - 2017. - Vol. 208, conf. 1. - DOI: 10.1088/1757899X/208/1/012010.

17. Ostroumov G. A. Free convection under the condition of the internal problem. - Washington : NACA Technical Memorandum 1407, National Advisory Committee for Aeronautics, 1958.

18. Birikh R. V. Thermocapillary convection in a horizontal layer of liquid // J. Appl. Mech. Tech. Phys. - 1966. - Vol. 7, no. 3. - P. 43-44.

19. Сидоров А. Ф. О двух классах решений уравнений механики жидкости и газа и их связи с теорией бегущих волн // Прикл. механика и технич. физика. - 1989. - № 2. - С. 34-40.

20. Aristov S. N., Prosviryakov E. Yu. A new class of exact solutions for three-dimensional thermal diffusion equations // Theor. Found. Chem. Eng. - 2016. - Vol. 50, no. 3. - P. 286-293. DOI: $10.1134 / \mathrm{S} 0040579516030027$.

21. Aristov S. N., Frik P. G. Nonlinear effects of the Ekman layer on the dynamics of largescale eddies in shallow water // J. Appl. Mech. Tech. Phys. - 1991. - Vol. 32, no. 2. - P. 189-194.

22. Ингель Л. Х., Аристов С. Н. Класс точных решенийнелинейных задач о термических циркуляциях, связанных с объемным тепловыделением в атмосфере // Тр. ин-та эксперим. метеорол. - 1991. - № 27 (162). - С. 142-157.

23. Аристов С. Н., Шварц К. Г. Вихревые течения адвективной природы во вращающемся слое жидкости. - Пермь : Перм. гос. ун-т., 2006. - 155 с.

24. Аристов С. Н., Шварц К. Г. Вихревые течения в тонких слоях жидкости. - Киров : Вятск. гос. ун-т, 2011. - 207 с.

25. Aristov S. N., Shvarts K. G. Advective flow in a rotating liquid film // J. Appl. Mech. Tech. Phys. - 2016. - Vol. 57, no. 1. - P. 188-194. - DOI: 10.1134/S0021894416010211.

26. Аристов С. Н., Просвиряков Е. Ю. О слоистых течениях плоской свободной конвекции // Нелин. динам. - 2013. - Т. 9, № 4. - С. 651-657. - DOI: 10.20537/nd1304004.

27. Андреев В. К. Решения Бириха уравнений конвекции и некоторые его обобщения // Красноярск : Ин-т вычисл. мех. СО РАН, препринт № 1-10, 2010. - 68 с.

28. Aristov S. N., Prosviryakov E. Yu., Spevak L. F. Unsteady-state Bénard-Marangoni convection in layered viscous incompressible flows // Theor. Found. Chem. Eng. - 2016. - Vol. 50, no. 2. P. 132-141. DOI: 10.1134/S0040579516020019.

29. Mathematical Models of Convection / V. K.Andreev, Ya. A. Gaponenko, O. N. Goncharova, V. V. Pukhnachev. - Berlin-Boston : De Gryuter Publ., 2012. - 417 p.

30. Pukhnachev V. V. Group-theoretical methods in the convection problems // Application of Mathematics in Technical and Natural Sciences / ed. by M. D. Todorov and C. I. Christov. Melwille, NY : American Institute of Physics, CP 1404, 2011. - P. 31-42.

31. Пухначев В. В. Нестационарные аналогии решения Бириха // Известия АлтГУ. - 2011. №. 1-2. - C. 62-69.

32. Andreev V. K., Bekezhanova V. B. Stability of non-isothermal fluids (Review) // J. Appl. Mech. Tech. Phys. - 2013. - Vol. 54, no. 2. - P. 171-184. - DOI: 10.1134/S0021894413020016.

33. Andreev V. K., Stepanova I. V. Unidirectional flows of binary mixtures within the framework of the Oberbeck-Boussinesq model // Fluid Dyn. - 2016. - Vol. 51, no. 2. - P. 136-147. DOI: $10.1134 /$ S0015462816020022.

34. Goncharova O. N., Kabov O. A. Gravitational-thermocapillary convection of fluid in the horizontal layer in co-current gas flow // Dokl. Phys. - 2009. - Vol. 54, no. 5. - P. 242-247. DOI: $10.1134 / \mathrm{S} 1028335809050061$.

35. Goncharova O. N., Rezanova E. V. Example of an exact solution of the stationary problem of two-layer flows with evaporation at the interface // J. Appl. Mech. Tech. Phys. - 2014. - Vol. 55, no. 2. - P. 247-257. - DOI: 10.1134/S0021894414020072. 
http://dream-journal.or"

36. Birikh R. V., Pukhnachev V. V. An axial convective flow in a rotating tube with a longitudinal temperature gradient // Dokl. Phys. - 2011. - Vol. 56, no. 1. - P. 47-52. DOI: $10.1134 / \mathrm{S} 1028335811010095$.

37. Birikh R. V., Pukhnachev V. V., Frolovskaya O. A. Convective flow in a horizontal channel with non-Newtonian surface rheology under time-dependent longitudinal temperature gradient // Fluid Dyn. - 2015. - Vol. 50, no. 1. - P. 173-179. - DOI: 10.1134/S0015462815010172.

38. Рыжков И. И. Термодиффузия в смесях: уравнения, симметрии, решения и их устойчивость. - Новосибирск : СО РАН, 2013. - 200 с. 